\title{
RATE OF CONVERGENCE OF SOLUTIONS OF RATIONAL DIFFERENCE EQUATION OF SECOND ORDER
}

\author{
S. KALABUŠIĆ AND M. R. S. KULENOVIĆ \\ Received 13 August 2003 and in revised form 7 October 2003
}

We investigate the rate of convergence of solutions of some special cases of the equation $x_{n+1}=\left(\alpha+\beta x_{n}+\gamma x_{n-1}\right) /\left(A+B x_{n}+C x_{n-1}\right), n=0,1, \ldots$, with positive parameters and nonnegative initial conditions. We give precise results about the rate of convergence of the solutions that converge to the equilibrium or period-two solution by using Poincaré's theorem and an improvement of Perron's theorem.

\section{Introduction and preliminaries}

We investigate the rate of convergence of solutions of some special types of the secondorder rational difference equation

$$
x_{n+1}=\frac{\alpha+\beta x_{n}+\gamma x_{n-1}}{A+B x_{n}+C x_{n-1}}, \quad n=0,1, \ldots,
$$

where the parameters $\alpha, \beta, \gamma, A, B$, and $C$ are positive real numbers and the initial conditions $x_{-1}, x_{0}$ are arbitrary nonnegative real numbers.

Related nonlinear second-order rational difference equations were investigated in [2, $5,6,7,8,9,10]$. The study of these equations is quite challenging and is in rapid development.

In this paper, we will demonstrate the use of Poincaré's theorem and an improvement of Perron's theorem to determine the precise asymptotics of solutions that converge to the equilibrium.

We will concentrate on three special cases of $(1.1)$, namely, for $n=0,1, \ldots$,

$$
\begin{aligned}
x_{n+1} & =\frac{B}{x_{n}}+\frac{C}{x_{n-1}}, \\
x_{n+1} & =\frac{p x_{n}+x_{n-1}}{q x_{n}+x_{n-1}}, \\
x_{n+1} & =\frac{p x_{n}+x_{n-1}}{q+x_{n-1}},
\end{aligned}
$$

Copyright (C) 2004 Hindawi Publishing Corporation Advances in Difference Equations 2004:2 (2004) 121-139 2000 Mathematics Subject Classification: 39A10, 39A11 URL: http://dx.doi.org/10.1155/S168718390430806X 
where all the parameters are assumed to be positive and the initial conditions $x_{-1}, x_{0}$ are arbitrary positive real numbers.

In [7], the second author and Ladas obtained both local and global stability results for (1.2), (1.3), and (1.4) and found the region in the space of parameters where the equilibrium solution is globally asymptotically stable. In this paper, we will precisely determine the rate of convergence of all solutions in this region by using Poincaré's theorem and an improvement of Perron's theorem.

We will show that the asymptotics of solutions that converge to the equilibrium depends on the character of the roots of the characteristic equation of the linearized equation evaluated at the equilibrium. The results on asymptotics of (1.2), (1.3), and (1.4) will show all the complexity of the asymptotics of the general equation (1.1).

Here we give some necessary definitions and results that we will use later.

Let $I$ be an interval of real numbers and let $f \in C^{1}[I \times I, I]$. Let $\bar{x} \in I$ be an equilibrium point of the difference equation

$$
x_{n+1}=f\left(x_{n}, x_{n-1}\right), \quad n=0,1, \ldots,
$$

that is, $\bar{x}=f(\bar{x}, \bar{x})$.

Let

$$
s=\frac{\partial f}{\partial u}(\bar{x}, \bar{x}), \quad t=\frac{\partial f}{\partial v}(\bar{x}, \bar{x})
$$

denote the partial derivatives of $f(u, v)$ evaluated at an equilibrium $\bar{x}$ of (1.5). Then the equation

$$
y_{n+1}=s y_{n}+t y_{n-1}, \quad n=0,1, \ldots
$$

is called the linearized equation associated with (1.5) about the equilibrium point $\bar{x}$.

Theorem 1.1 (linearized stability). (a) If both roots of the quadratic equation

$$
\lambda^{2}-s \lambda-t=0
$$

lie in the open unit disk $|\lambda|<1$, then the equilibrium $\bar{x}$ of (1.5) is locally asymptotically stable.

(b) If at least one of the roots of (1.8) has an absolute value greater than one, then the equilibrium $\bar{x}$ of (1.5) is unstable.

(c) A necessary and sufficient condition for both roots of (1.8) to lie in the open unit disk $|\lambda|<1$ is

$$
|s|<1-t<2 \text {. }
$$

In this case, the locally asymptotically stable equilibrium $\bar{x}$ is also called a sink.

(d) A necessary and sufficient condition for both roots of (1.8) to have absolute values greater than one is

$$
|t|>1, \quad|s|<|1-t| .
$$

In this case, $\bar{x}$ is called a repeller. 
(e) A necessary and sufficient condition for one root of (1.8) to have an absolute value greater than one and for the other to have an absolute value less than one is

$$
s^{2}+4 t>0, \quad|s|>|1-t|
$$

In this case, the unstable equilibrium $\bar{x}$ is called a saddle point.

The set of points whose orbits converge to an attracting equilibrium point or, periodic point is called the "basin of attraction," see [1, page 128].

Definition 1.2. Let $\mathbf{T}$ be a map on $\mathbb{R}^{2}$ and let $\mathbf{p}$ be an equilibrium point or a periodic point for $\mathbf{T}$. The basin of attraction of $\mathbf{p}$, denoted by $\mathscr{S}_{\mathbf{p}}$, is the set of points $\mathbf{x} \in \mathbb{R}^{2}$ such that $\left|\mathbf{T}^{k}(\mathbf{x})-\mathbf{T}^{k}(\mathbf{p})\right| \rightarrow 0$, as $k \rightarrow \infty$, that is,

$$
\mathscr{B}_{\mathbf{p}}=\left\{\mathbf{x} \in \mathbb{R}^{2}:\left|\mathbf{T}^{k}(\mathbf{x})-\mathbf{T}^{k}(\mathbf{p})\right| \longrightarrow 0, \text { as } k \longrightarrow \infty\right\}
$$

where $|\cdot|$ denotes any norm in $\mathbb{R}^{2}$.

We now give the definitions of positive and negative semicycles of a solution of (1.5) relative to an equilibrium point $\bar{x}$.

A positive semicycle of a solution $\left\{x_{n}\right\}$ of (1.5) consists of a "string" of terms $\left\{x_{l}\right.$, $\left.x_{l+1}, \ldots, x_{m}\right\}$, all greater than or equal to the equilibrium $\bar{x}$, with $l \geq-1$ and $m \leq \infty$ and such that either $l=-1$ or $l>-1, x_{l-1}<\bar{x}$, and either $m=\infty$ or $m<\infty, x_{m+1}<\bar{x}$. A negative semicycle of a solution $\left\{x_{n}\right\}$ of (1.5) consists of a string of terms $\left\{x_{l}, x_{l+1}, \ldots, x_{m}\right\}$, all less than the equilibrium $\bar{x}$, with $l \geq-1$ and $m \leq \infty$ and such that either $l=-1$ or $l>$ $-1, x_{l-1} \geq \bar{x}$, and either $m=\infty$ or $m<\infty, x_{m+1} \geq \bar{x}$.

The next theorem is a slight modification of the result obtained in $[7,9]$.

Theorem 1.3. Assume that

$$
f:[0, \infty) \times[0, \infty) \longrightarrow[0, \infty)
$$

is a continuous function satisfying the following properties:

(a) there exist $L$ and $U, 0<L<U$, such that

$$
f(L, L) \geq L, \quad f(U, U) \leq U,
$$

and $f(x, y)$ is nondecreasing in $x$ and $y$ in $[L, U]$;

(b) the equation

$$
f(x, x)=x
$$

has a unique positive solution in $[L, U]$.

Then (1.5) has a unique equilibrium $\bar{x} \in[L, U]$ and every solution of (1.5) with initial values $x_{-1}, x_{0} \in[L, U]$ converges to $\bar{x}$. 
124 Rate of convergence of rational difference equation

Proof. Set

$$
m_{0}=L, \quad M_{0}=U,
$$

and for $i=1,2, \ldots$, set

$$
M_{i}=f\left(M_{i-1}, M_{i-1}\right), \quad m_{i}=f\left(m_{i-1}, m_{i-1}\right) .
$$

Now observe that for each $i \geq 0$,

$$
\begin{gathered}
m_{0} \leq m_{1} \leq \cdots \leq m_{i} \leq \cdots \leq M_{i} \leq \cdots \leq M_{1} \leq M_{0}, \\
m_{i} \leq x_{k} \leq M_{i} \quad \text { for } k \geq 2 i+1 .
\end{gathered}
$$

Now the proof follows as the proof of [7, Theorem 1.4.8].

The next two theorems give precise information about the asymptotics of linear nonautonomous difference equations. Consider the scalar $k$ th-order linear difference equation

$$
x(n+k)+p_{1}(n) x(n+k-1)+\cdots+p_{k}(n) x(n)=0,
$$

where $k$ is a positive integer and $p_{i}: \mathbb{Z}^{+} \rightarrow \mathbb{C}$ for $i=1, \ldots, k$. Assume that

$$
q_{i}=\lim _{k \rightarrow \infty} p_{i}(n), \quad i=1, \ldots, k
$$

exist in $\mathbb{C}$. Consider the limiting equation of (1.19):

$$
x(n+k)+q_{1} x(n+k-1)+\cdots+q_{k} x(n)=0 .
$$

Then the following results describe the asymptotics of solutions of (1.19). See [4, 3, $11]$.

Theorem 1.4 (Poincaré's theorem). Consider (1.19) subject to condition (1.20). Let $\lambda_{1}, \ldots$, $\lambda_{k}$ be the roots of the characteristic equation

$$
\lambda^{k}+q_{1} \lambda^{k-1}+\cdots+q_{k}=0
$$

of the limiting equation (1.21), and suppose that

$$
\left|\lambda_{i}\right| \neq\left|\lambda_{j}\right| \quad \text { for } i \neq j
$$

If $x(n)$ is a solution of (1.19), then either $x(n)=0$ for all large $n$ or there exists an index $j \in\{1, \ldots, k\}$ such that

$$
\lim _{n \rightarrow \infty} \frac{x(n+1)}{x(n)}=\lambda_{j}
$$


The related results were obtained by Perron, and one of Perron's results was improved by Pituk, see [11].

Theorem 1.5. Suppose that (1.20) holds. If $x(n)$ is a solution of (1.19), then either $x(n)=0$ eventually or

$$
\limsup _{n \rightarrow \infty}\left(\left|x_{j}(n)\right|\right)^{1 / n}=\left|\lambda_{j}\right|
$$

where $\lambda_{1}, \ldots, \lambda_{k}$ are the (not necessarily distinct) roots of the characteristic equation (1.22).

2. Rate of convergence of $x_{n+1}=\left(B / x_{n}\right)+\left(C / x_{n-1}\right)$

Equation (1.2) has a unique equilibrium point $\bar{x}=\sqrt{B+C}$. The linearized equation associated with (1.2) about $\bar{x}$ is

$$
z_{n+1}+\frac{B}{B+C} z_{n}+\frac{C}{B+C} z_{n-1}=0, \quad n=0,1, \ldots
$$

This equation was considered in [7], where the method of full limiting sequences was used to prove that the equilibrium is globally asymptotically stable for all values of parameters $B$ and $C$. Here, we want to establish the rate of this convergence. The characteristic equation

$$
\lambda^{2}+\frac{B}{B+C} \lambda+\frac{C}{B+C}=0, \quad n=0,1, \ldots,
$$

that corresponds to (2.1) has roots

$$
\lambda_{ \pm}=\frac{-B \pm \sqrt{B^{2}-4 C(B+C)}}{2(B+C)} .
$$

THeOREm 2.1. All solutions of (1.2) which are eventually different from the equilibrium satisfy the following.

(i) If the condition

$$
C<\frac{B}{2(1+\sqrt{2})}
$$

holds, then

$$
\lim _{n \rightarrow \infty} \frac{x_{n+1}-\bar{x}}{x_{n}-\bar{x}}=\lambda_{+} \quad \text { or } \quad \lim _{n \rightarrow \infty} \frac{x_{n+1}-\bar{x}}{x_{n}-\bar{x}}=\lambda_{-},
$$

where $\lambda_{ \pm}$are the real roots given by (2.3).

In particular, all solutions of (1.2) oscillate.

(ii) If the condition

$$
C=\frac{B}{2(1+\sqrt{2})}
$$


holds, then

$$
\limsup _{n \rightarrow \infty}\left(\left|x_{n}-\bar{x}\right|\right)^{1 / n}=\frac{B}{2(B+C)} .
$$

(iii) If the condition

$$
C>\frac{B}{2(1+\sqrt{2})}
$$

holds, then

$$
\limsup _{n \rightarrow \infty}\left(\left|x_{n}-\bar{x}\right|\right)^{1 / n}=\left|\lambda_{ \pm}\right|
$$

where $\lambda_{ \pm}$are the complex roots given by (2.3).

Proof. We have

$$
x_{n+1}-\bar{x}=\frac{B}{x_{n}}+\frac{C}{x_{n-1}}-\bar{x}=-\frac{B}{x_{n} \bar{x}}\left(x_{n}-\bar{x}\right)-\frac{C}{x_{n-1} \bar{x}}\left(x_{n-1}-\bar{x}\right) .
$$

Set $e_{n}=x_{n}-\bar{x}$. Then we obtain

$$
e_{n+1}+p_{n} e_{n}+q_{n} e_{n-1}=0
$$

where

$$
p_{n}=\frac{B}{x_{n} \bar{x}}, \quad q_{n}=\frac{C}{x_{n-1} \bar{x}} .
$$

Since the equilibrium is a global attractor, we obtain

$$
\lim _{n \rightarrow \infty} p_{n}=\frac{B}{B+C}, \quad \lim _{n \rightarrow \infty} q_{n}=\frac{C}{B+C}
$$

Thus, the limiting equation of (1.2) is the linearized equation (2.1) whose characteristic equation is (2.2). The discriminant of this equation is given by

$$
D=B^{2}-4 C(B+C)=(B-2 \sqrt{C(B+C)})(B+2 \sqrt{C(B+C)}) .
$$

Conditions (2.4), (2.6), and (2.8) are the conditions for $D>0, D=0$, and $D<0$, respectively.

Now, statement (i) follows as an immediate consequence of Poincaré's theorem and statements (ii) and (iii) follow as the consequences of Theorem 1.5. Finally, the statement on oscillatory solutions follows from the asymptotic estimate (2.5) and the fact that in the case $D>0$ both roots $\lambda_{ \pm}<0$. 


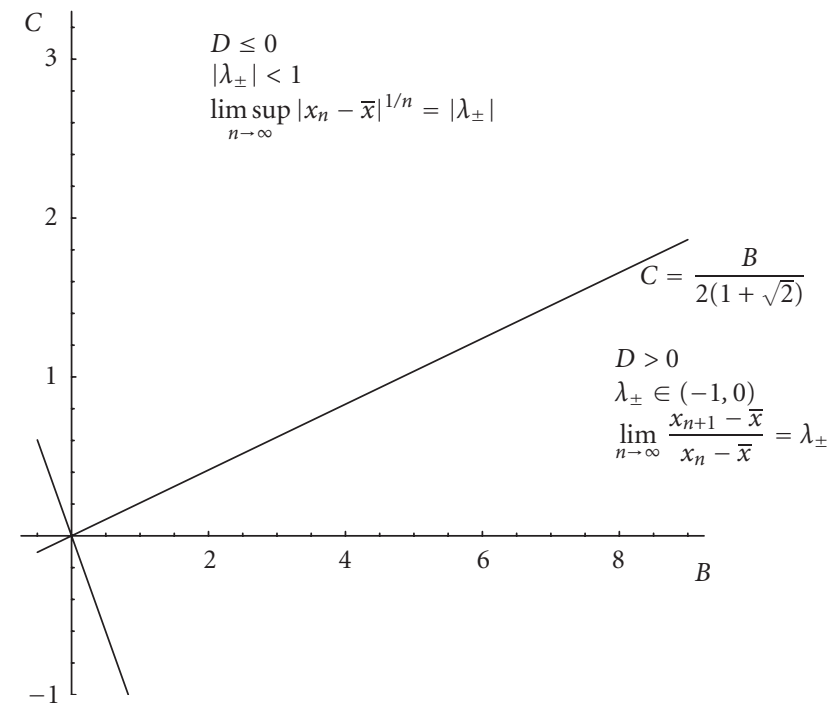

Figure 2.1. Regions for the different asymptotic behavior of solutions of (1.2).

Figure 2.1 visualizes the regions for the different asymptotic behavior of solutions of (1.2).

3. Rate of convergence of $x_{n+1}=\left(p x_{n}+x_{n-1}\right) /\left(q x_{n}+x_{n-1}\right)$

Equation (1.3) was studied in detail in $[7,10]$, where we have found the region of parameters for which the equilibrium is globally asymptotically stable and the region where the equation has a unique period-two solution which is locally asymptotically stable.

3.1. Rate of convergence of the equilibrium. Equation (1.3) has a unique equilibrium point

$$
\bar{x}=\frac{p+1}{q+1} .
$$

To avoid the trivial case, we assume that $p \neq q$.

The linearized equation associated with (1.3) about $\bar{x}$ is

$$
z_{n+1}-\frac{p-q}{(p+1)(q+1)} z_{n}+\frac{p-q}{(p+1)(q+1)} z_{n-1}=0, \quad n=0,1, \ldots
$$

The characteristic equation

$$
\lambda^{2}-\frac{p-q}{(p+1)(q+1)} \lambda+\frac{p-q}{(p+1)(q+1)}=0
$$


128 Rate of convergence of rational difference equation

has the roots

$$
\lambda_{ \pm}=\frac{p-q \pm \sqrt{(q-p)(4 p q+3 p+5 q+4)}}{2(p+1)(q+1)} .
$$

This equation was considered in detail in $[7,10]$, where it was proved that the equilibrium is globally asymptotically stable for values of parameters $p$ and $q$ that satisfy

$$
p<q<\frac{3 p+1}{1-p}
$$

or

$$
\frac{p-1}{p+3}<q<p
$$

Here, we want to establish the rate of convergence.

THeorem 3.1. All solutions of (1.3) which are eventually different from the equilibrium satisfy the following.

(i) If condition (3.5) holds, then (2.5) follows, where $\lambda_{ \pm}$are given by (3.4).

(ii) If condition (3.6) holds, then

$$
\limsup _{n \rightarrow \infty}\left(\left|x_{n}-\bar{x}\right|\right)^{1 / n}=\left|\lambda_{ \pm}\right|
$$

where $\lambda_{ \pm}$are given by (3.4).

Proof. We have

$$
x_{n+1}-\bar{x}=\frac{p x_{n}+x_{n-1}}{q x_{n}+x_{n-1}}-\bar{x}=\frac{p-q \bar{x}}{q x_{n}+x_{n-1}}\left(x_{n}-\bar{x}\right)+\frac{1-\bar{x}}{q x_{n}+x_{n-1}}\left(x_{n-1}-\bar{x}\right) .
$$

Set $e_{n}=x_{n}-\bar{x}$. Then we obtain

$$
e_{n+1}-p_{n} e_{n}-q_{n} e_{n-1}=0,
$$

where

$$
p_{n}=\frac{p-q \bar{x}}{q x_{n}+x_{n-1}}, \quad q_{n}=\frac{1-\bar{x}}{q x_{n}+x_{n-1}} .
$$

As the equilibrium is a global attractor, we obtain

$$
\lim _{n \rightarrow \infty} p_{n}=\frac{p-q \bar{x}}{(1+q) \bar{x}}=\frac{p-q}{(p+1)(q+1)}, \quad \lim _{n \rightarrow \infty} q_{n}=\frac{q-p}{(p+1)(q+1)} .
$$

Thus, the limiting equation of (1.3) is the linearized equation (3.2).

Now, statement (i) follows as an immediate consequence of Poincaré's theorem and statement (ii) follows as a consequence of Theorem 1.5. 


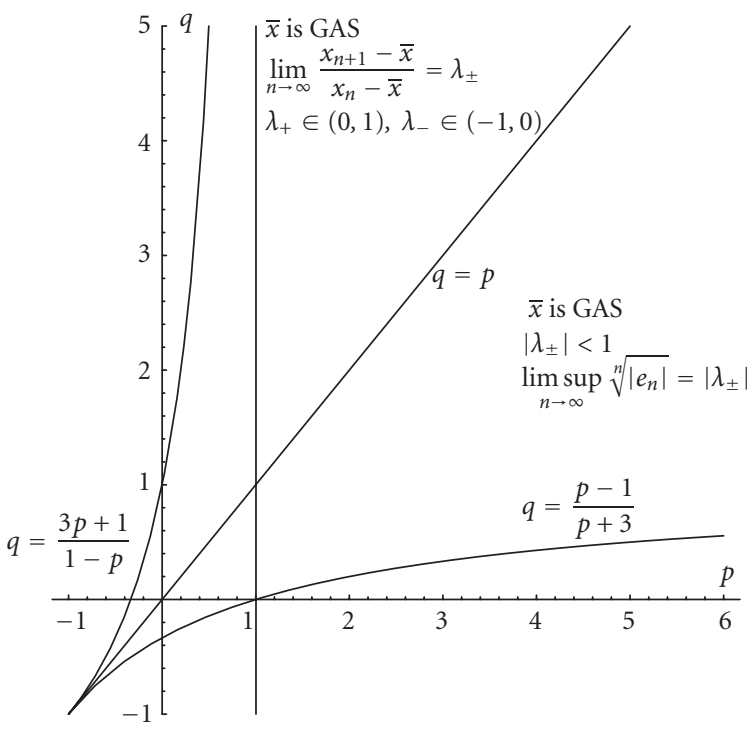

Figure 3.1. Regions for the asymptotic behavior of solutions of (1.3).

Figure 3.1 visualizes the regions for the different asymptotic behavior of solutions of (1.3)

3.2. Rate of convergence of period-two solutions. Assume that

$$
q>1+3 p+p q
$$

or equivalently,

$$
p<1, \quad q>\frac{1+3 p}{1-p}
$$

Then (1.3) possesses the prime period-two solution ..., $\Phi, \Psi, \Phi, \Psi, \ldots$, see $[7,10]$. Without loss of generality, we assume that $\Phi<\Psi$. Let $\left\{y_{n}\right\}_{n=-1}^{\infty}$ be a solution of (1.3). Then the following identities are true:

$$
\begin{aligned}
& y_{n+1}-\Psi=(q-p) \frac{y_{n-1} \Phi-y_{n} \Psi}{\left(y_{n-1}+q y_{n}\right)(\Psi+q \Phi)}, \\
& y_{n+1}-\Phi=(q-p) \frac{y_{n-1} \Psi-y_{n} \Phi}{\left(y_{n-1}+q y_{n}\right)(\Phi+q \Psi)} .
\end{aligned}
$$

The following lemma is now a direct consequence of (3.14).

Lemma 3.2. Assume that condition (3.12) holds. Let $\left\{y_{n}\right\}_{n=-1}^{\infty}$ be a solution of (1.3). Then the following statements are true.

(i) If, for some $N \geq 0, y_{N-1}>\Psi, y_{N}<\Phi$, then $y_{N+1}>\Psi$.

(ii) If, for some $N \geq 0, y_{N-1}<\Phi, y_{N}>\Psi$, then $y_{N+1}<\Phi$. 
(iii) Every solution $\left\{y_{n}\right\}_{n=-1}^{\infty}$ of (1.3) with initial conditions that satisfy

$$
y_{-1}>\Psi, \quad y_{0}<\Phi \quad \text { or } \quad y_{-1}<\Phi, \quad y_{0}>\Psi
$$

oscillates with semicycles of length one. More precisely, such a solution oscillates about the strip $[\Phi, \Psi]$ with semicycles of length one.

Proof. (i) The proof follows from

$$
y_{N+1}-\Psi>(q-p) \Phi \frac{y_{N-1}-\Psi}{\left(y_{N-1}+q y_{N}\right)(\Psi+q \Phi)} .
$$

(ii) Similarly, the proof is an immediate consequence of

$$
y_{N+1}-\Phi<(q-p) \Psi \frac{y_{N-1}-\Phi}{\left(y_{N-1}+q y_{N}\right)(\Phi+q \Psi)} .
$$

(iii) The proof follows from (i) and (ii).

Now, we will combine our results for semicycles to identify solutions which converge to the period-two solution.

Theorem 3.3. Assume that condition (3.12) holds. Then every solution of (1.3) with initial conditions

$$
y_{-1}>1, \quad y_{0}<\frac{p}{q}
$$

or

$$
y_{-1}<\frac{p}{q}, \quad y_{0}>1
$$

converges to the period-two solution ..., $\Phi, \Psi, \Phi, \Psi, \ldots$, where $\Phi<\Psi$ are the roots of

$$
t^{2}-(1-p) t+\frac{p(1-p)}{q-1}=0 .
$$

Proof. We will prove the statements in the case (3.18). The proof of the second case is similar.

It is known that for $q>p$, which holds in view of $(3.12)$, the interval $[p / q, 1]$ is an invariant and attracting interval for (1.3), and that $y_{n} \in[p / q, 1], n \geq 1$, for every solution $\left\{y_{n}\right\}$ of $(1.3)$, see $[7,10]$. In particular, $p / q<\Phi<\Psi<1$. Then Lemma 3.2 implies that

$$
y_{2 k+1}>\Psi, \quad y_{2 k+2}<\Phi, \quad k=0,1, \ldots
$$

Further, by using the identity

$$
y_{n+1}-y_{n-1}=\frac{y_{n-1}\left(1-y_{n-1}\right)+q y_{n}\left(p / q-y_{n-1}\right)}{y_{n-1}+q y_{n}},
$$


we obtain

$$
y_{1}<y_{-1}, \quad y_{2}>y_{0} .
$$

Now, by using the monotonic character of the function $f(x, y)=(p x+y) /(q x+y)$ which decreases in $x$ and increases in $y$ for $q>p$, we have

$$
y_{3}=f\left(y_{2}, y_{1}\right)<f\left(y_{0}, y_{-1}\right)=y_{1}, \quad y_{4}=f\left(y_{3}, y_{2}\right)>f\left(y_{1}, y_{0}\right)=y_{2} \text {. }
$$

By using induction, we obtain

$$
\cdots<y_{5}<y_{3}<y_{1}, \quad y_{2}<y_{4}<y_{6}<\cdots .
$$

Thus, we conclude that the sequence $\left\{y_{2 k+1}\right\}_{k=0}^{\infty}$ is nonincreasing and $y_{2 k+1}>\Psi$, which implies that

$$
\lim _{k \rightarrow \infty} y_{2 k+1}=L \geq \Psi \text {. }
$$

Likewise, the sequence $\left\{y_{2 k+2}\right\}_{k=0}^{\infty}$ is nondecreasing and $y_{2 k+2}<\Phi$, which implies that

$$
\lim _{k \rightarrow \infty} y_{2 k+2}=l \leq \Phi .
$$

In view of the uniqueness of the prime period-two solution, we have

$$
L=\Psi, \quad l=\Phi,
$$

which completes the proof of the theorem.

The last theorem gives us information about the basin of attraction of the prime period-two solutions, which we denote by $\mathbf{B}_{2}$. We have shown that

$$
\left\{(x, y): x>1, y<\frac{p}{q}\right\} \cup\left\{(x, y): x<\frac{p}{q}, y>1\right\} \subset \mathbf{B}_{2} .
$$

Now, we will combine our results for convergence to period-two solution of (1.3) to obtain the rate of convergence.

By using identities (3.14) and Theorem 3.3, we obtain

$$
y_{2 k+1}-\Psi=\frac{(q-p) \Phi}{A_{k}}\left(y_{2 k-1}-\Psi\right)-\frac{(q-p) \Psi}{A_{k}}\left(y_{2 k}-\Phi\right),
$$

where

$$
A_{k}=(\Psi+q \Phi)\left(y_{2 k-1}+q y_{2 k}\right)
$$

and

$$
y_{2 k}-\Phi=\frac{(q-p) \Psi}{B_{k}}\left(y_{2 k-2}-\Phi\right)-\frac{(q-p) \Phi}{B_{k}}\left(y_{2 k-1}-\Psi\right)
$$


132 Rate of convergence of rational difference equation

where

$$
B_{k}=(\Phi+q \Psi)\left(y_{2 k-2}+q y_{2 k-1}\right) \text {. }
$$

By using (3.32), identity (3.30) implies

$$
y_{2 k+2}-\Phi=\frac{(q-p) \Phi}{B_{k}}\left(\frac{\Psi}{\Phi}+\frac{B_{k}}{A_{k}}+\frac{(q-p) \Psi}{A_{k}}\right)\left(y_{2 k}-\Phi\right)-\frac{(q-p)^{2} \Phi \Psi}{A_{k} B_{k}}\left(y_{2 k-2}-\Phi\right)
$$

Set

$$
e_{k}=y_{2 k}-\Phi
$$

Then (3.34) becomes

$$
e_{k+1}=c_{k} e_{k}+d_{k} e_{k-1}
$$

where

$$
c_{k}=\frac{(q-p) \Phi}{B_{k}}\left(\frac{\Psi}{\Phi}+\frac{B_{k}}{A_{k}}+\frac{(q-p) \Psi}{A_{k}}\right), \quad d_{k}=-\frac{(q-p)^{2} \Phi \Psi}{A_{k} B_{k}}
$$

with

$$
\begin{aligned}
& \lim _{k \rightarrow \infty} c_{k}=\frac{(1+2 p+p q)(q-1)(1-p)+p(q-p)}{(1-p)(q-p)(q-1)} \\
& \lim _{n \rightarrow \infty} d_{k}=-\frac{p}{(q-1)(1-p)}
\end{aligned}
$$

Thus, the limiting equation of (3.36) is

$$
e_{k+1}-\frac{(1+2 p+p q)(q-1)(1-p)+p(q-p)}{(1-p)(q-p)(q-1)} e_{k}+\frac{p}{(q-1)(1-p)} e_{k-1}=0
$$

The characteristic equation of (3.39) is

$$
\lambda^{2}-\frac{(1+2 p+p q)(q-1)(1-p)+p(q-p)}{(1-p)(q-p)(q-1)} \lambda+\frac{p}{(q-1)(1-p)}=0
$$

Note that (3.40) is the characteristic equation of second iterate of the map that corresponds to (1.3), evaluated at the period-two solution, see [7, page 115]. 
The discriminant of (3.40) is

$$
D=\left(\frac{(1+2 p-p q)(q-1)(1-p)+p(q-p)}{(p-1)(q-p)(q-1)}\right)^{2}-4 \frac{p}{(1-p)(q-1)}
$$

If condition (3.12) holds, then $D$ can be greater or less than zero.

By using (3.32), we obtain

$$
e_{k+1}-a_{k} e_{k}+b_{k} e_{k-1}=0, \quad k=0,1, \ldots
$$

where

$$
\begin{gathered}
e_{k}=y_{2 k-1}-\Psi, \\
a_{k}=\frac{(q-p) \Psi}{A_{k}}\left(\frac{\Phi}{\Psi}+\frac{(q-p) \Phi}{B_{k}}+\frac{A_{k-1}}{B_{k}}\right), \\
b_{k}=\frac{(q-p)^{2} \Phi \Psi}{A_{k} B_{k}},
\end{gathered}
$$

with

$$
\lim _{n \rightarrow \infty} a_{n}=\lim _{n \rightarrow \infty} c_{n}, \quad \lim _{n \rightarrow \infty} b_{n}=\lim _{n \rightarrow \infty} d_{n} .
$$

Thus, the limiting equation of (3.42) is (3.39). Using Poincaré's theorem and Theorem 1.5 , we obtain the following result which describes the precise asymptotics of convergence to a period-two solution.

Theorem 3.4. Assume that condition (3.13) holds. Then every solution $\left\{x_{n}\right\}_{n=-1}^{\infty}$ of (1.3), which is eventually different from a period-two solution, that converges to a period-two solution satisfies one of the following two asymptotic relations:

(a)

$$
\begin{array}{lll}
\lim _{n \rightarrow \infty} \frac{x_{2 n+1}-\Psi}{x_{2 n-1}-\Psi}=\lambda_{+} & \text {or } & \lim _{n \rightarrow \infty} \frac{x_{2 n+1}-\Psi}{x_{2 n-1}-\Psi}=\lambda_{-}, \\
\lim _{n \rightarrow \infty} \frac{x_{2 n+2}-\Phi}{x_{2 n}-\Phi}=\lambda_{+} & \text {or } & \lim _{n \rightarrow \infty} \frac{x_{2 n+2}-\Phi}{x_{2 n}-\Phi}=\lambda_{-},
\end{array}
$$

when $D>0$;

(b)

$$
\limsup _{n \rightarrow \infty}\left(\left|x_{2 n+1}-\Psi\right|\right)^{1 /(2 n+1)}=\limsup _{n \rightarrow \infty}\left(\left|x_{2 n}-\Phi\right|\right)^{1 / 2 n}=\left|\lambda_{ \pm}\right|
$$

when $D \leq 0$, where $\lambda_{ \pm}$are solutions of (3.40). Here, $D$ is given by (3.41). 
134 Rate of convergence of rational difference equation

4. Rate of convergence of $x_{n+1}=\left(p x_{n}+x_{n-1}\right) /\left(q+x_{n-1}\right)$

Equation (1.4) was investigated in detail in $[7,9]$. Here, we assume that $p$ and $q$ are positive parameters.

Equation (1.4) has two equilibrium points $\bar{x}=0$ and $\bar{x}=p+1-q$ if $p+1>q$.

The linearized equation of (1.4) at the zero equilibrium is

$$
z_{n+1}-\frac{p}{q} z_{n}-\frac{1}{q} z_{n-1}=0
$$

with characteristic equation

$$
\lambda^{2}-\frac{p}{q} \lambda-\frac{1}{q}=0
$$

The solutions of (4.2) are

$$
\lambda_{ \pm}=\frac{p \pm \sqrt{p^{2}+4 q}}{2 q} .
$$

The linearized equation of (1.4) at the positive equilibrium $\bar{x}$ is

$$
z_{n+1}-\frac{p}{p+1} z_{n}-\frac{q-p}{p+1} z_{n-1}=0
$$

with characteristic equation

$$
\lambda^{2}-\frac{p}{p+1} \lambda-\frac{q-p}{p+1}=0
$$

The solutions of (4.5) are

$$
\lambda_{ \pm}=\frac{1}{2(p+1)}(p \pm \sqrt{(4 q(p+1)-p(3 p+4))}) .
$$

Now, we give two results that describe precisely the asymptotics of the solutions that converge to either zero or the positive equilibrium.

Theorem 4.1. Assume that $p+1 \leq q$. Then the zero equilibrium of (1.4) is globally asymptotically stable and

$$
\lim _{n \rightarrow \infty} \frac{x_{n+1}}{x_{n}}=\lambda_{+} \quad \text { or } \quad \lim _{n \rightarrow \infty} \frac{x_{n+1}}{x_{n}}=\lambda_{-},
$$

for every solution $x_{n}$ of (1.4) which is eventually different from the zero equilibrium. Here, $\lambda_{ \pm}$are given by (4.6).

Proof. Global asymptotic stability was established in $[7,9]$.

Now, we can represent (1.4) in the form

$$
x_{n+1}=\frac{p x_{n}+x_{n-1}}{q+x_{n-1}}=a_{n} x_{n}+b_{n} x_{n-1},
$$


where

$$
a_{n}=\frac{p}{q+x_{n-1}}, \quad b_{n}=\frac{1}{q+x_{n-1}},
$$

with

$$
\lim _{n \rightarrow \infty} a_{n}=\frac{p}{q}, \quad \lim _{n \rightarrow \infty} b_{n}=\frac{1}{q}
$$

Thus, the limiting equation is exactly the linearized equation (4.1), and an application of Poincaré's theorem completes the proof of the theorem.

Now, we assume that $p+1>q$.

Theorem 4.2. Assume that $p+1>q$ and $x_{-1}+x_{0}>0$. Then the positive equilibrium of (1.4) is globally asymptotically stable and the solutions exhibit one of the following two types of asymptotic behavior.

(i) Suppose that the condition

$$
q>\frac{p(3 p+4)}{4(p+1)}
$$

is satisfied. Then every solution $\left\{x_{n}\right\}$ of (1.4) which is eventually different from the equilibrium satisfies one of the following two limit relations:

$$
\lim _{n \rightarrow \infty} \frac{x_{n+1}-\bar{x}}{x_{n}-\bar{x}}=\lambda_{+} \quad \text { or } \quad \lim _{n \rightarrow \infty} \frac{x_{n+1}-\bar{x}}{x_{n}-\bar{x}}=\lambda_{-},
$$

where $\lambda_{ \pm}$are the real roots given by (4.6).

If $p=q$, then every solution $\left\{x_{n}\right\}$ of (1.4) which is eventually different from the equilibrium satisfies one of the following two limit relations:

$$
\lim _{n \rightarrow \infty} \frac{x_{n+1}-\bar{x}}{x_{n}-\bar{x}}=\lambda_{ \pm}
$$

where $\lambda_{ \pm}$is either 0 or $p /(p+1)$.

(ii) Suppose that the condition

$$
q \leq \frac{p(3 p+4)}{4(p+1)}
$$

is satisfied. Then every solution $\left\{x_{n}\right\}$ of (1.4) which is eventually different from the equilibrium satisfies

$$
\limsup _{n \rightarrow \infty}\left|x_{n}-\bar{x}\right|^{1 / n}=\left|\lambda_{ \pm}\right|
$$

where $\lambda_{ \pm}$are the complex roots given by (4.6). 
Proof. The proof of global asymptotic stability was given in $[7,9]$. Here, we want to correct the proof in the case where $p<q$. As we have shown in $[7,9]$, in this case, the interval $(0,1)$ is invariant and attracting in the sense that every positive solution eventually enters and remains in the interval $(0,1)$. Now, in the interval $(0,1)$, the function

$$
f(u, v)=\frac{p u+v}{q+v}
$$

is increasing in both arguments and it has a unique equilibrium. Now, we check condition (a) of Theorem 1.3. We try to determine $L, U, 0<L<p+1-q<U$, that satisfy the conditions $f(L, L) \geq L$ and $f(U, U) \leq U$. We obtain

$$
\frac{(p+1) L}{q+L} \geq L, \quad \frac{(p+1) U}{q+U} \leq U,
$$

which are always satisfied for $0<L<p+1-q<U$. By Theorem 1.3, every solution of (1.4) converges to the positive equilibrium, and since this equilibrium is locally asymptotically stable, it is also globally asymptotically stable.

Now, we will establish results on the rate of convergence to the positive equilibrium. We have

$$
\begin{gathered}
x_{n+1}-\bar{x}=\frac{p x_{n}+x_{n-1}}{q+x_{n-1}}-\bar{x}=\frac{p}{q+x_{n-1}}\left(x_{n}-\bar{x}\right)+\frac{q-p}{q+x_{n-1}}\left(x_{n-1}-\bar{x}\right), \\
e_{n+1}-p_{n} e_{n}-q_{n} e_{n-1}=0,
\end{gathered}
$$

where

$$
e_{n}=x_{n}-\bar{x}, \quad p_{n}=\frac{p}{q+x_{n-1}}, \quad q_{n}=\frac{q-p}{q+x_{n-1}} .
$$

As the positive equilibrium is a global attractor, we obtain

$$
\lim _{n \rightarrow \infty} p_{n}=\frac{p}{p+1}, \quad \lim _{n \rightarrow \infty} q_{n}=\frac{q-p}{p+1} .
$$

Thus, the limiting equation of (1.4) is the linearized equation (4.4).

Now, statement (i) follows as an immediate consequence of Poincaré's theorem and statement (ii) follows as a consequence of Theorem 1.5. Conditions (4.11) and (4.14) are actually conditions for the characteristic equation (4.5) to have two real distinct roots and to have double or complex conjugate roots, respectively.

Figure 4.1 visualizes the regions for the different asymptotic behavior of solutions of (1.4). 


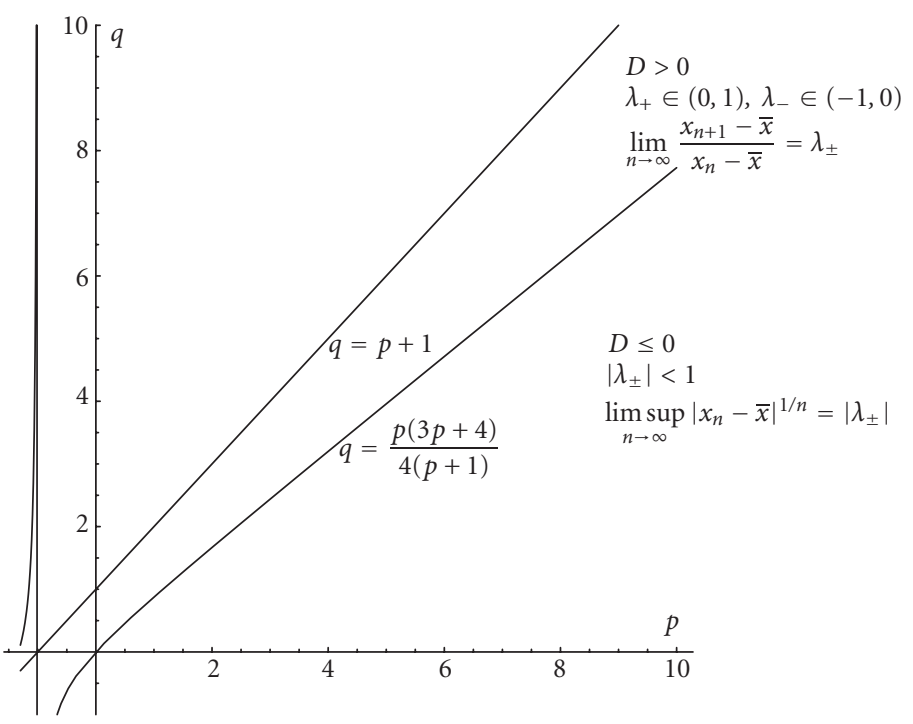

Figure 4.1. Regions for the asymptotic behavior of solutions of (1.4).

\section{Rate of convergence of (1.1)}

Consider (1.1) where the parameters $\alpha, \beta, \gamma, A, B$, and $C$ are nonnegative real numbers and the initial conditions $x_{-1}$ and $x_{-2}$ are arbitrary nonnegative real numbers such that

$$
A+B x_{n}+C x_{n-1}>0 \quad \forall n \geq 0 .
$$

The equilibrium point of (1.1) is

$$
\bar{x}=\frac{\alpha+\bar{x}(\beta+\gamma)}{A+\bar{x}(B+C)} .
$$

Then we have

$$
x_{n+1}-\bar{x}=a_{n}\left(x_{n}-\bar{x}\right)+b_{n}\left(x_{n-1}-\bar{x}\right), \quad n=0,1, \ldots,
$$

where

$$
\begin{aligned}
& a_{n}=\frac{\beta A-\alpha B+\bar{x}(\beta C-\gamma B)}{\left(A+B x_{n}+C x_{n-1}\right)(A+\bar{x}(B+C))}, \\
& b_{n}=\frac{\gamma A-\alpha C+\bar{x}(\gamma B-\beta C)}{\left(A+B x_{n}+C x_{n-1}\right)(A+\bar{x}(B+C))} .
\end{aligned}
$$

Set $x_{n}-\bar{x}=e_{n}$. Then (5.3) becomes

$$
e_{n+1}-a_{n} e_{n}-b_{n} e_{n-1}=0, \quad n=0,1, \ldots,
$$


where

$$
a_{n} \longrightarrow \frac{\beta A-\alpha B+\bar{x}(\beta C-\gamma B)}{(A+\bar{x}(B+C))^{2}}, \quad b_{n} \longrightarrow \frac{\gamma A-\alpha C+\bar{x}(\gamma B-\beta C)}{(A+\bar{x}(B+C))^{2}}, \quad n \rightarrow \infty .
$$

The limiting equation associated with (5.5) is

$$
e_{n+1}-\frac{\beta A-\alpha B+\bar{x}(\beta C-\gamma B)}{(A+\bar{x}(B+C))^{2}} e_{n}-\frac{\gamma A-\alpha C+\bar{x}(\gamma B-\beta C)}{(A+\bar{x}(B+C))^{2}} e_{n-1}=0, \quad n=0,1, \ldots
$$

The characteristic equation of (5.7) is

$$
\lambda^{2}-\frac{\beta A-\alpha B+\bar{x}(\beta C-\gamma B)}{(A+\bar{x}(B+C))^{2}} \lambda-\frac{\gamma A-\alpha C+\bar{x}(\gamma B-\beta C)}{(A+\bar{x}(B+C))^{2}}=0
$$

which is exactly the characteristic equation of the linearized equation of (1.1) evaluated at the positive equilibrium $\bar{x}$.

Using Poincaré's theorem and Theorem 1.5, we obtain the following result which describes the precise asymptotic behavior of solutions converging to the positive equilibrium.

Theorem 5.1. (i) If the discriminant of (5.8) is positive, then every solution $\left\{x_{n}\right\}$ of (1.1) which is eventually different from the equilibrium satisfies one of the two limit relations in (4.12), where $\lambda_{ \pm}$are the real roots of (5.8).

In particular, (1.1) has all oscillatory solutions if both roots $\lambda_{ \pm}$are negative, and has all solutions nonoscillatory if both roots $\lambda_{ \pm}$are positive.

(ii) If the discriminant of (5.8) is nonpositive, then every solution $\left\{x_{n}\right\}$ of (1.1) which is eventually different from the equilibrium satisfies (3.7), where $\lambda_{ \pm}$are the complex roots of (5.8).

\section{Acknowledgments}

The authors are grateful to the referees for numerous comments that improved the quality of the paper. The first author is on research leave from the Department of Mathematics, University of Sarajevo, Sarajevo, Bosnia and Herzegovina.

\section{References}

[1] K. T. Alligood, T. D. Sauer, and J. A. Yorke, Chaos. An Introduction to Dynamical Systems, Textbooks in Mathematical Sciences, Springer-Verlag, New York, 1997.

[2] H. El-Metwally, E. A. Grove, and G. Ladas, A global convergence result with applications to periodic solutions, J. Math. Anal. Appl. 245 (2000), no. 1, 161-170.

[3] S. N. Elaydi, An Introduction to Difference Equations, 2nd ed., Undergraduate Texts in Mathematics, Springer-Verlag, New York, 1999.

[4] - Recent developments in the asymptotics of difference equations, New Developments in Difference Equations and Applications (Taipei, 1997), Gordon and Breach, Amsterdam, 1999, pp. 161-181.

[5] C. H. Gibbons, M. R. S. Kulenović, and G. Ladas, On the recursive sequence $x_{n+1}=(\alpha+$ $\left.\beta x_{n-1}\right) /\left(\gamma+x_{n}\right)$, Math. Sci. Res. Hot-Line 4 (2000), no. 2, 1-11. 
[6] V. L. Kocić and G. Ladas, Global Behavior of Nonlinear Difference Equations of Higher Order with Applications, Mathematics and Its Applications, vol. 256, Kluwer Academic Publishers, Dordrecht, 1993.

[7] M. R. S. Kulenović and G. Ladas, Dynamics of Second Order Rational Difference Equations, Open Problems and Conjectures, Chapman \& Hall/CRC, Florida, 2002.

[8] M. R. S. Kulenović, G. Ladas, and N. R. Prokup, On the recursive sequence $x_{n+1}=\left(\alpha x_{n}+\right.$ $\left.\beta x_{n-1}\right) /\left(A+x_{n}\right)$, J. Differ. Equations Appl. 6 (2000), no. 5, 563-576.

[9] _ A rational difference equation, Comput. Math. Appl. 41 (2001), no. 5-6, 671-678.

[10] M. R. S. Kulenović, G. Ladas, and W. S. Sizer, On the recursive sequence $x_{n+1}=\left(\alpha x_{n}+\right.$ $\left.\beta x_{n-1}\right) /\left(\gamma x_{n}+\delta x_{n-1}\right)$, Math. Sci. Res. Hot-Line 2 (1998), no. 5, 1-16.

[11] M. Pituk, More on Poincaré's and Perron's theorems for difference equations, J. Difference Equ. Appl. 8 (2002), no. 3, 201-216.

S. Kalabušić: Department of Mathematics, University of Rhode Island, Kingston, RI 02881-0816, USA

E-mail address: senadak@pmf.unsa.ba

M. R. S. Kulenović: Department of Mathematics, University of Rhode Island, Kingston, RI 028810816, USA

E-mail address: kulenm@math.uri.edu 